\title{
Serum neurofilament light chain (sNfL) values in a large cross-sectional population of children with asymptomatic to moderate COVID-19
}

\author{
Tobias Geis ${ }^{1}$. Susanne Brandstetter ${ }^{1,2}$. Antoaneta A. Toncheva ${ }^{1} \cdot$ Otto Laub ${ }^{3}$. Georg Leipold ${ }^{4} \cdot$ Ralf Wagner $^{5}$. \\ Michael Kabesch $^{1,2} \cdot$ Severin Kasser $^{6} \cdot$ Jens Kuhle ${ }^{7} \cdot$ Sven Wellmann ${ }^{1}$ (D) CoKiBa Study group
}

Received: 16 January 2021 / Revised: 8 April 2021 / Accepted: 9 April 2021 / Published online: 23 April 2021

(c) The Author(s) 2021

\begin{abstract}
Background Serum neurofilament light chain (sNfL) is an established biomarker of neuro-axonal damage in multiple neurological disorders. Raised sNfL levels have been reported in adults infected with pandemic coronavirus disease 2019 (COVID-19). Levels in children infected with COVID-19 have not as yet been reported.

Objective To evaluate whether sNfL is elevated in children contracting COVID-19.

Methods Between May 22 and July 22, 2020, a network of outpatient pediatricians in Bavaria, Germany, the Coronavirus antibody screening in children from Bavaria study network (CoKiBa), recruited healthy children into a cross-sectional study from two sources: an ongoing prevention program for 1-14 years, and referrals of 1-17 years consulting a pediatrician for possible infection with severe acute respiratory syndrome coronavirus 2 (SARS-CoV-2). We determined sNfL levels by single molecule array immunoassay and SARS-CoV-2 antibody status by two independent quantitative methods.

Results Of the 2652 included children, 148 (5.6\%) were SARS-CoV-2 antibody positive with asymptomatic to moderate COVID-19 infection. Neurological symptoms-headache, dizziness, muscle aches, or loss of smell and taste—were present in $47 / 148$ cases (31.8\%). Mean sNfL levels were $5.5 \mathrm{pg} / \mathrm{ml}$ (SD 2.9) in the total cohort, 5.1 (SD 2.1) pg/ml in the children with SARS-CoV-2 antibodies, and 5.5 (SD 3.0) pg/ml in those without. Multivariate regression analysis revealed age-but neither antibody status, antibody levels, nor clinical severity - as an independent predictor of sNfL. Follow-up of children with pediatric multisystem inflammatory syndrome $(n=14)$ showed no association with sNfL.

Conclusions In this population study, children with asymptomatic to moderate COVID-19 showed no neurochemical evidence of neuronal damage.
\end{abstract}

Keywords COVID-19 $\cdot$ Children $\cdot$ Brain $\cdot$ Neurology $\cdot$ Neurofilament

Jens Kuhle and Sven Wellmann contributed equally.

The members of CoKiBa Study group are listed in acknowledgements.

Sven Wellmann

sven.wellmann@ukr.de

1 University Children's Hospital Regensburg (KUNO), Hospital St. Hedwig of the Order of St. John, University of Regensburg, Steinmetzstr. 1-3, 93049 Regensburg, Germany

2 Research and Development Campus Regensburg (WECARE), at the Hospital St. Hedwig of the Order of St. John, University of Regensburg, Regensburg, Germany

3 Pediatric Office Dr. Laub, Rosenheim, Germany

4 Pediatric Office Dr. Leipold, Regensburg, Germany
5 Institute of Medical Microbiology and Hygiene, Molecular Microbiology (Virology), University of Regensburg, Regensburg, Germany

6 Department of Pediatric Hematology and Oncology, University Children's Hospital Basel (UKBB), University of Basel, Basel, Switzerland

7 Neurology Clinic and Policlinic, MS Center and Research Center for Clinical Neuroimmunology and Neuroscience Basel, University Hospital Basel, University of Basel, Basel, Switzerland 


\section{Introduction}

Multiple differences have emerged between adults and children in the clinical manifestations of severe acute respiratory syndrome coronavirus 2 (SARS-CoV-2) infection, with notable respect to severity, outcome, and neurological involvement [1-3]. In addition, in children with coronavirus disease 2019 (COVID-19), a multisystem hyperinflammatory syndrome has recently been identified, termed pediatric multiorgan immune syndrome (PMIS), which shares many clinical features with Kawasaki disease [4], including a presumed immune-mediated post-infectious etiology [5].

Neurofilament light chain (NfL), a cytoplasmic protein exclusively expressed in central and peripheral nervous system neurons, has recently become established as a specific biomarker of neuroaxonal damage [6]. Raised levels of serum NfL (sNfL) have been found in numerous acute and chronic neurologic diseases, including in adults with mild-to-moderate and severe COVID-19 [7, 8]; levels in pediatric COVID-19 and PMIS have not as yet been reported.

Our aim in this study was to measure SARS-CoV-2 antibody and SNfL levels in a large pediatric population to determine the impact of COVID-19 on neuronal integrity.

\section{Materials and methods}

\section{Study design and population}

The Coronavirus antibodies in Kids from Bavaria (CoKiBa) study group collected samples for this cross-sectional study between May 22 and July 222020 in three regions of Southern Germany, which were strongly affected by the first wave of the pandemic. Using a sliding window approach CoKiBa invited participation by the parents of all the 1-14 years, they were scheduled to see in 2020 as part of a prevention program. They also invited any child, including siblings older than 14 years, who with their parents' consent wished to take part. Exclusion criteria for participating in this $\mathrm{SNfL}$ study were preexisting chronic or congenital diseases. All data were collected using an online self-administered parental questionnaire. Entries were fully anonymized and only accessible to participants using an individual code on the Qnome platform (www.qnome.eu), as previously detailed [9]. The study was approved by the University of Regensburg institutional review board (20-1865-101), and written informed consent was obtained from the parents.

\section{SARS-CoV-2 antibody tests}

The specific antibody response to SARS-CoV-2 was evaluated using two test kits: the commercially available licensed qualitative Elecsys ${ }^{\circledR}$ Anti-SARS-CoV-2 assay (Roche Diagnostics, Rotkreuz, Switzerland; https://diagn ostics.roche.com) and a validated in-house ELISA [10]. The Elecsys ${ }^{\circledR}$ Anti-SARS-CoV-2 assay does not discriminate between the antibody type(s) present and can detect $\operatorname{IgA}, \operatorname{IgM}$, and $\operatorname{IgG}$. The test is based on a recombinant nucleocapsid $(\mathrm{N})$ antigen and has a cutoff value of 1.0 (S/Co). The in-house ELISA based on the SARS-CoV-2 $\mathrm{S}$-protein receptor-binding domain quantifies total $\mathrm{IgG}$ and has a cutoff value of $1.0(\mathrm{~S} / \mathrm{Co})$. All S/Co results $<1.0$ were considered negative. S/Co antibody responses $\geq 100$ in the Elecsys ${ }^{\circledR}$ Anti-SARS-CoV-2 assay were defined as strong and participants were invited for clinical follow-up for PMIS.

\section{sNfL analysis}

sNfL was measured at the same timepoint as Anti-SARSCoV-2 using the digital NF-light ${ }^{\mathrm{TM}}$ single molecule array assay on the HD-X Analyzer (Quanterix, Lexington, MA), as described elsewhere [6].

\section{Statistical analyses}

Descriptive statistics were calculated for the entire sample and both subgroups (children with and without SARS-CoV-2 antibodies). As sNfL values ranged widely, with considerable numbers of outliers, logarithmic transformation was used to normalize distributions. We used multivariable linear regression (MLR) models adjusted for age and sex to analyze associations between SARS-Cov-2 antibody status, antibody levels, PMIS symptoms and neurologic symptoms on the one hand and SNfL levels on the other. We performed all analyses using IBM SPSS Statistics 24.

\section{Results}

A total of 2934 children were recruited, of whom 2832 (96.5\%) were successfully tested for SARS-CoV-2 antibodies and had complete personal data. Samples sufficient for sNfL analysis were obtained from 2687 participants, 35 individuals were excluded due to preexisting chronic or congenital disease, including epilepsy $(n=12)$, cerebral palsy $(n=3)$, cystic fibrosis or chromosomal/genetic diseases $(n=10)$, inborn metabolic or endocrinological disorders $(n=5)$, oncologic, or inflammatory diseases $(n=5)$. Of the 
Table 1 Symptoms and severity in SARS-Cov-2 antibody-positive children

\begin{tabular}{lc}
\hline Symptoms and severity, $n(\%)$ & $\begin{array}{l}\text { Antibody-positive } \\
n=148\end{array}$ \\
\hline None at all & $38(25.7)$ \\
No neurologic symptom & $101(68.2)$ \\
Headache & $33(22.3)$ \\
Dizziness & $6(4.0)$ \\
Muscle aches & $24(16.2)$ \\
Loss of smell & $6(4.0)$ \\
Loss of taste & $7(4.7)$ \\
Hospitalization for COVID-19 & $2(1.3)$ \\
\hline
\end{tabular}

Table 2 Population characteristics stratified by SARS-CoV-2 antibody status

\begin{tabular}{llll}
\hline & $\begin{array}{l}\text { All } \\
n=2652\end{array}$ & $\begin{array}{l}\text { Antibody-positive } \\
n=148\end{array}$ & $\begin{array}{l}\text { Antibody- } \\
\text { negative } \\
n=2504\end{array}$ \\
\hline $\begin{array}{l}\text { Sex (female), } n \\
(\%)\end{array}$ & $1280(48.3)$ & $73(49.3)$ & $1207(48.2)$ \\
$\begin{array}{l}\text { Age (years), M } \\
(\mathrm{SD})\end{array}$ & $7.1(3.8)$ & $7.5(4.0)$ & $7.1(3.8)$ \\
sNfL, M (SD) & $5.5(2.9)$ & $5.1(2.1)$ & $5.5(3.0)$ \\
\hline
\end{tabular}

$s N f L$ Serum neurofilament light chain

remaining 2652 participants, 148 (5.6\%) were SARS-CoV-2 antibody-positive by virtue of an above-cutoff result in at least one of the two antibody tests. Table 1 lists the neurologic symptoms in SARS-CoV-2-positive participants. There were no significant age or sex differences between the antibody-positive and negative subgroups (Table 2). In 64 (43\%) children the timepoint of COVID-19 onset was available as at last one SARS-CoV-2 PCR-positive nasopharyngeal swab was recorded from the children themselves $(n=25)$ or their parents $(n=39)$, which was on average 85 days ( $\min 5$, max 118, IQR 59-93 days) prior to blood sampling for SARS$\mathrm{CoV}-2$ antibodies and SNfL analysis.

The mean sNfL level in the total cohort was $5.5 \mathrm{pg} / \mathrm{ml}$ (SD 2.9); mean levels in the antibody-positive and negative subgroups were 5.1 (SD 2.1) pg/ml and 5.5 (SD 3.0) $\mathrm{pg} / \mathrm{ml}$. Multivariable MLR analysis revealed no significant association between SARS-CoV-2 antibody status and sNfL (Fig. 1).

In the antibody-positive subgroup, we tested for correlation between antibody and sNfL levels separately for the Elecsys ${ }^{\circledR}$ results $(n=130$; Fig. 2a) and ELISA results ( $n=147$; Fig. $2 b$ ). MLR revealed no significant association in either analysis.

Children with SARS-CoV-2 antibody levels $>100$ were followed up for possible PMIS: 14 of the 50 children were

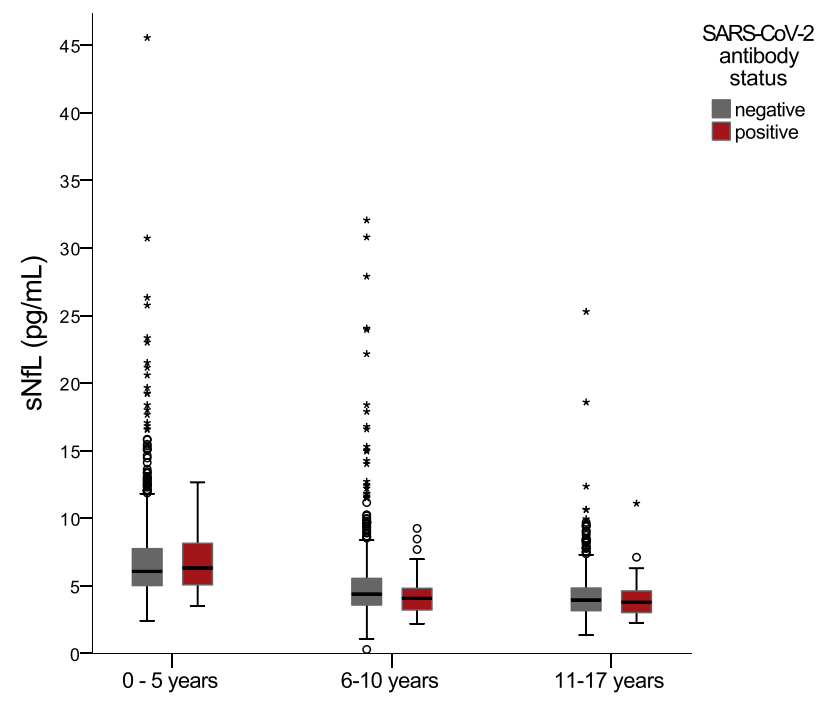

Fig. 1 Association of SARS-CoV-2 antibody status (negative/positive) with sNfL. SARS-CoV-2 antibody status is not significantly associated with sNfL after adjusting for age and sex (linear regression analysis: $\mathrm{B}=-0.052, \mathrm{SE}(\mathrm{B})=0.030 ; P=.088 . n=2651)$

diagnosed with one or more PMIS-compatible symptoms. However, we found no association between PMIS symptoms and sNfL levels (regression coefficient $\mathrm{B}=0.22$; standardized regression coefficient beta $=0.099, P=0.144$ ).

Nor did we find any association, after adjusting for age and sex, between sNfL levels and the time elapsing from COVID-19 onset to blood sampling for SARS-CoV-2 antibodies (regression coefficient $\mathrm{B}<0.01$; standardized regression coefficient beta $=0.007, P=0.948$ ).

\section{Discussion}

Our population study found no increase in the levels of sNfL, a highly specific biomarker for neuronal damage, in children with asymptomatic to moderate SARS-CoV-2 infection. There was no association with $\mathrm{sNfL}$ even in children with extremely high antibody levels, neurologic symptoms, or symptoms consistent with PMIS.

Early in the pandemic sustained SNfL elevation reflecting neurologic involvement was described in adults with severe COVID-19 admitted to intensive care [8, 11]. More surprisingly, raised levels were also found in adults with mild-to-moderate COVID-19 who were either neurologically asymptomatic or who exhibited only minor neurologic symptoms [7]. Our result is in line with findings in young adults $<35$ years of age and suggests that children are less susceptible to neurologic involvement than older adults [1, 7]. Further evidence of age dependency is provided by the higher rate of neurologic complications in older COVID-19 patients [11]. 

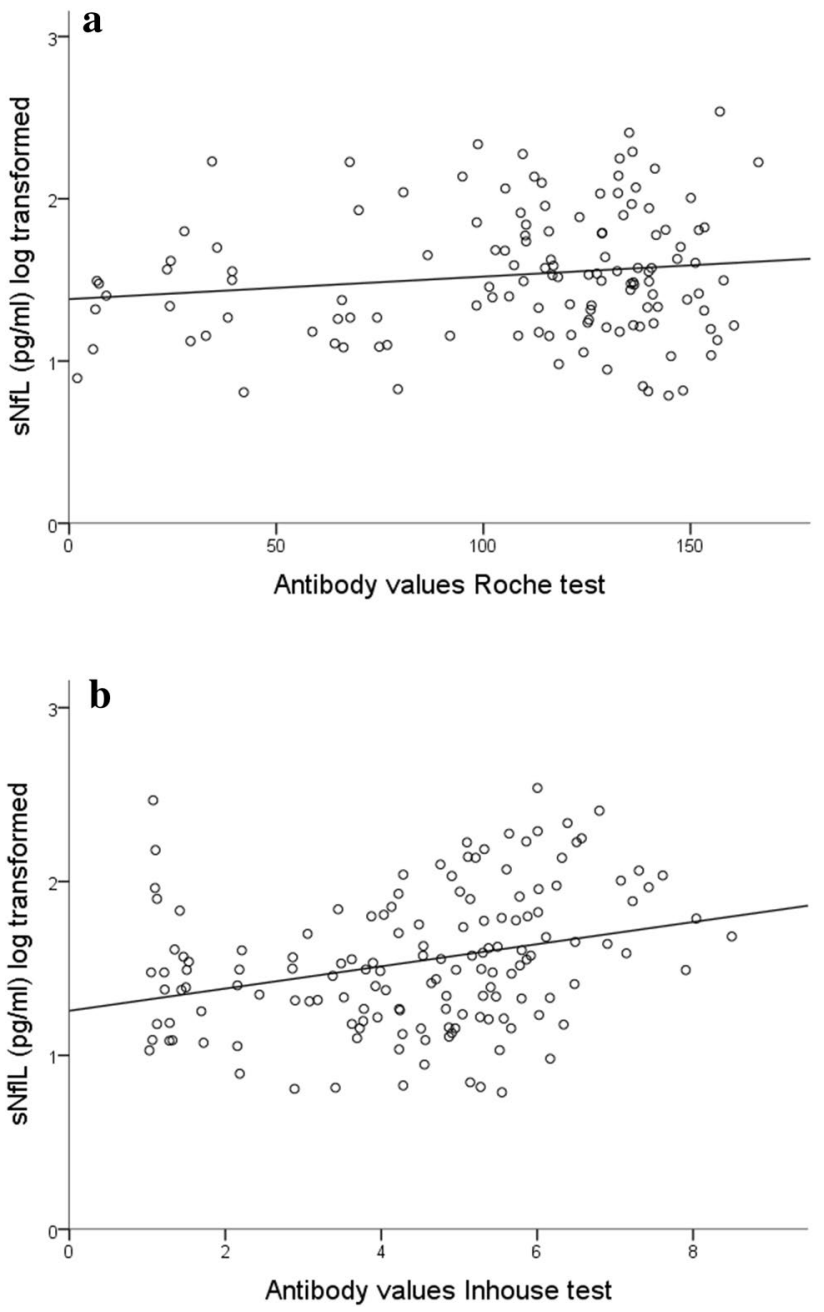

Fig. 2 Association of SARS-CoV-2 antibody levels with sNfL. Antibody levels in the Roche test $(\mathbf{a} ; n=130)$ and inhouse test $(\mathbf{b} ; n=147)$ are not significantly associated with sNfL after adjusting for age and sex [linear regression analysis: $\mathbf{a} \mathrm{B}=0.001, \mathrm{SE}(\mathrm{B})=0.001 ; P=.310$. b $\mathrm{B}=0.022$, $\mathrm{SE}(\mathrm{B})=0.015 ; P=.144]$. Antibody levels: all participants with a positive test result $>1$

PMIS presents with a wide spectrum of signs and symptoms [4] and is thought to occur as an exaggerated autoimmune response to infection between one to more than 9 weeks after COVID-19 [12]. A characteristic pattern of PMIS-associated cytokine storm and immune response has been identified [12], prompting the hypothesis that a specific hyperinflammatory response to SARS-CoV-2 infection causes the neuronal damage in PMIS [5, 13]. Fortunately, all the PMIS children in our study made a full neurologic recovery without sequelae. The absence of sNfL elevation in children confirms that neurologic involvement in postCOVID-19 PMIS is rather benign and the outcome is better than in neurologically impacted adults with COVID-19.
The study has several limitations. First, access to SARSCoV-2 testing was variable by region and limited particularly during the early phase of the pandemic. Second, the number of PMIS patients in our study is limited $(n=14)$ and PMIS severity low; therefore, additional studies are warranted to investigate neuroaxonal integrity in those children. Third, in all participants, blood collection was done only at one timepoint; thus, the here reported results do not reflect sNfL dynamics which have higher predictive power than single point of time determinations, e.g., in Alzheimer's disease or children with poor neurodevelopmental outcome $[14,15]$.

In conclusion, based on the deployment of $\mathrm{SNfL}$ as a biomarker for neuronal damage in a large pediatric population with asymptomatic to moderate COVID-19, there is no evidence that SARS-CoV-2 impacts neuroaxonal integrity in infected children.

Acknowledgements The CoKiBa Study group are (in alphabetical order): Bettina Aichholzer, Georg Mair, Michaela Wruk, Imke Reischl; paediatric office; Dr. Aichholzer, Dr. Mair, Dr. Wruk, Reischl; Bad Endorf; Germany. Andreas Ambrosch; Institute of Laboratory Medicine, Microbiology and Hygiene, Hospital of the Order of St. John, Regensburg, Germany. David Antos; paediatric office; Dr. Antos; Traunstein; Germany. Stephan von Koskull, Christian Becker; paediatric office; Dr. von Koskul, Becker; Bad Aibling; Germany. Elisabeth Beer, Hubert Schirmer; paediatric office; Dr. Beer, Dr. Schirmer; Marktredwitz; Germany. Georg Birkinger; paediatric office; Dr. Birkinger; Wasserburg am Inn; Germany. Andreas Blueml; paediatric office; Blueml; Trostberg; Germany. Heike Buntrock-Döpke, Research and Development Campus Regensburg (WECARE) at the Hospital St. Hedwig of the Order of St. John, Regensburg, Germany. Mona Castrop; paediatric office; Dr. Castrop; Regensburg; Germany. Jost Dieckerhoff; paediatric office; Dr. Dieckerhoff; Rosenheim; Germany. Renate Eichhorn; paediatric office; Dr. Eichhorn; Regensburg; Germany. Dominik Ewald; Pediatric office Drs. Heuschmann \& Ewald, Regensburg and Regenstauf, Germany. Gudrun Fleck, Alfred Heihoff; paediatric office; Dr. Fleck, Dr. Heihoff; Regensburg; Germany. Jürgen Geuder; paediatric office; Dr. Geuder; Freilassing; Germany. Jens Grombach; paediatric office; Dr. Grombach; Neuoetting; Germany. Peter Gutdeutsch, Florian Segerer; paediatric office; Dr. Gutdeutsch, Dr. Segerer; Regensburg; Germany. Thomas Habash, Sonja Habash; paediatric office; Dres. Habash; Cham; Germany. Susanne Harner, University Childrens' Hospital Regensburg (KUNO) at the Hospital St. Hedwig of the Order of St. John University of Regensburg, Regensburg, Germany. Christoph Herbst; paediatric office; Dr. Herbst; Edling; Germany. Daniela Heuschmann; paediatric office; Dr. Heuschmann; Regensburg/Regenstauf; Germany. Meike Hofmann; paediatric office; Hofmann; Mitterteich; Germany. Michael Horn; paediatric office; Dr. Horn; Schoenau am Chiemsee; Germany. Birgit Jork-Kaeferlein, Monika Schwarz, Reinhard Hopfner; paediatric office; Dr. Jork-Kaeferlein, Dr. Schwarz, Dr. Hopfner; Prien; Germany. Guido Judex, Bastian Baumgartner, Monika Corbacioglu, Sabrina Lindner, Bettina Meinel, Alena Bauer, Hannes Löw, Annamaria Szulagyi-Kovacs; paediatric office; Dr. Judex, Dr. Baumgartner, Dr. Corbacioglu, Dr. Lindner, Dr. Meinel, Bauer, Löw, Szulagyi-Kovacs; Regensburg; Germany. Sarah Laub, Pediatric Office Dr. Laub, Rosenheim, Germany. Annegret Klein; paediatric office; Dr. Klein; Oberaudorf; Germany. Cosima Koering; paediatric office; Dr. Koering; Altoetting; Germany. Niclas Landvogt, Claudia Soehngen, Karin Rasp, Gudrun Schick-Niedermeier; paediatric office; Dr. Landvogt, Dr. Soehngen; Traunreut; Germany. Marinus Laub; paediatric office; Laub; Rosenheim; Germany. Otto Laub; paediatric office; Laub; Rosenheim; Germany. Georg Leipold, 
Petra Schmid-Seibold; paediatric office; Dr. Leipold Schmid-Seibold; Regensburg; Germany. Johannes Pawlak, Michaela Reitz; paediatric office; Dr. Pawlak, Dr. Reitz; Rosenheim; Germany. David Peterhoff, Institute of Medical Microbiology and Hygiene, Molecular Microbiology (Virology), University of Regensburg, Regensburg, Germany. Georg Puchner; paediatric office; Dr. Puchner; Regensburg; Germany. Christiane Razeghi, Stefan Razeghi; paediatric office; Dres. Razeghi; Miesbach; Germany. Christine Rehe, Klaus Rehe; paediatric office; Dres. Rehe; Kolbermoor; Germany. Matthias Scheffel, Ludwig Kaesbauer; paediatric office; Dr. Scheffel, Dr. Kaesbauer; Muehldorf; Germany. Roland Schmid, Michael Strobelt; paediatric office; Dr. Schmid, Dr. Strobelt; Bruckmuehl; Germany. Nina Schoetzau; paediatric office; Dr. Schoetzau; Miesbach; Germany. Andrea Schweiger-Kabesch; University Children's Hospital Regensburg (KUNO) at the Hospital St. Hedwig of the Order of St. John and the University Hospital, University of Regensburg, Regensburg, Germany. Marko Senjor; paediatric office; Dr. Senjor; Wasserburg; Germany. Michael Sperlich; paediatric office; Dr. Sperlich; Ampfing; Germany. Guenter Theuerer, Guenter Steidle; paediatric office; Dr. Theurer, Dr. Steidle; Traunstein; Germany. German Tretter; paediatric office; Dr. Tretter; Altenstadt an der Waldnaab; Germany. Victor von Arnim; paediatric office; Dr. von Arnim; Roding; Germany. Marlene Volz-Fleckenstein; paediatric office; Dr. Volz-Fleckenstein; Regensburg; Germany. Sebastian Einhauser, Patrick Neckermann; Institute of Medical Microbiology and Hygiene, Molecular Microbiology (Virology), University of Regensburg, Regensburg, Germany. Natascha Borchers; University Children's Hospital Regensburg (KUNO) at the Hospital St. Hedwig of the Order of St. John, University of Regensburg, Regensburg, Germany. Elisangela Santos-Valente, Parastoo Kheiroddin; University Children's Hospital Regensburg (KUNO) at the Hospital St. Hedwig of the Order of St. John, University of Regensburg, Regensburg, Germany. Patricia Schöberl; Research and Development Campus Regensburg (WECARE) at the Hospital St. Hedwig of the Order of St. John, Regensburg, Germany. Jakob Niggel; Maganamed Limited, Regensburg, Germany. Klaus Überla; Institute of Clinical and Molecular Virology, FAU Erlangen-Nuremberg and Universitätsklinikum Erlangen, Erlangen, Germany. Stephan Gerling; University Children's Hospital Regensburg (KUNO), University of Regensburg, Regensburg, Germany.

Authors contribution All authors listed contributed to the study conception and design, with main lead by Michael Kabesch and Sven Wellmann. Material preparation, data collection and analysis were performed by Tobias Geis, Susanne Brandstetter, Antoaneta A. Toncheva, Otto Laub, Georg Leipold, Ralf Wagner, Severin Kasser and Jens Kuhle. The members of the CoKiBa Study group comprise medical doctors in outpatient settings who recruited the study participants and performed the study visits, listed in alphabetical order below. The first draft of the manuscript was written by Tobias Geis and Sven Wellmann and all authors commented on previous versions of the manuscript. All authors read and approved the final manuscript.

Funding Open Access funding enabled and organized by Projekt DEAL. This work was funded by the Bavarian State Ministry of Science and the Arts (StMWK; Prospective Covid-19 Cohort Tirschenreuth [TiKoCo19] grant to RW), and by institutional grants. Roche Diagnostics provided Elecsys Anti-SARS-CoV-2 immunoassay tests free of charge.

\section{Availability of data and material On request.}

\section{Declarations}

Conflicts of interest All authors declare that they have no competing financial or personal interests.
Ethics approval The Ethics Committee of the University of Regensburg approved the study (file-number: 20-1865-101).

Consent to participate Written informed consent was obtained from the parents.

Consent for publication Written informed consent was obtained from the parents.

Open Access This article is licensed under a Creative Commons Attribution 4.0 International License, which permits use, sharing, adaptation, distribution and reproduction in any medium or format, as long as you give appropriate credit to the original author(s) and the source, provide a link to the Creative Commons licence, and indicate if changes were made. The images or other third party material in this article are included in the article's Creative Commons licence, unless indicated otherwise in a credit line to the material. If material is not included in the article's Creative Commons licence and your intended use is not permitted by statutory regulation or exceeds the permitted use, you will need to obtain permission directly from the copyright holder. To view a copy of this licence, visit http://creativecommons.org/licenses/by/4.0/.

\section{References}

1. Kim Y, Walser SA, Asghar SJ, Jain R, Mainali G, Kumar A (2020) A comprehensive review of neurologic manifestations of COVID19 and management of pre-existing neurologic disorders in children. J Child Neurol https://doi.org/10.1177/0883073820968995

2. Ludvigsson JF (2020) Systematic review of COVID-19 in children shows milder cases and a better prognosis than adults. ActaPaediatr 109(6):1088-1095. https://doi.org/10.1111/apa.15270

3. Abdel-Mannan O, Eyre M, Löbel U et al (2020) Neurologic and radiographic findings associated with COVID-19 infection in children. JAMA Neurol https://doi.org/10.1001/jamaneurol.2020. 2687

4. Whittaker E, Bamford A, Kenny J et al (2020) Clinical characteristics of 58 children with a pediatric inflammatory multisystem syndrome temporally associated with SARS-CoV-2. JAMA 324(3):259-269. https://doi.org/10.1001/jama.2020.10369

5. Chen TH (2020) Neurological involvement associated with COVID-19 infection in children. J NeurolSci 418:117096. https:// doi.org/10.1016/j.jns.2020.117096

6. Khalil M, Teunissen CE, Otto M et al (2018) Neurofilaments as biomarkers in neurological disorders. Nat Rev Neurol 14(10):577589. https://doi.org/10.1038/s41582-018-0058-z

7. Ameres M, Brandstetter S, Toncheva AA et al (2020) Association of neuronal injury blood marker neurofilament light chain with mild-to-moderate COVID-19. J Neurol 267(12):3476-3478. https://doi.org/10.1007/s00415-020-10050-y

8. Kanberg N, Ashton NJ, Andersson LM et al (2020) Neurochemical evidence of astrocytic and neuronal injury commonly found in COVID-19. Neurology 95(12):e1754-e1759. https://doi.org/ 10.1212/WNL.0000000000010111

9. Brandstetter S, Toncheva AA, Niggel J et al (2019) KUNO-Kids birth cohort study: rationale, design, and cohort description. Mol Cell Pediatr 6(1):1. https://doi.org/10.1186/s40348-018-0088-z

10. Peterhoff D, Glück V, Vogel M et al (2020) A highly specific and sensitive serological assay detects SARS-CoV-2 antibody levels in COVID-19 patients that correlate with neutralization. Infection https://doi.org/10.1007/s15010-020-01503-7

11. Mao L, Jin H, Wang M et al (2020) Neurologic manifestations of hospitalized patients with coronavirus disease 2019 in Wuhan, 
China. JAMA Neurol 77(6):683-690. https://doi.org/10.1001/ jamaneurol.2020.1127

12. Belay ED, Abrams J, Oster ME et al (2021) Trends in geographic and temporal distribution of US children with multisystem inflammatory syndrome during the COVID-19 pandemic. JAMA Pediatr https://doi.org/10.1001/jamapediatrics.2021.0630

13. Consiglio CR, Cotugno N, Sardh F et al (2020) The immunology of multisystem inflammatory syndrome in children with COVID19. Cell 183(4):968-981.e7. https://doi.org/10.1016/j.cell.2020.09. 016
14. Preische O, Schultz SA, Apel A et al (2019) Serum neurofilament dynamics predicts neurodegeneration and clinical progression in presymptomatic Alzheimer's disease. Nat Med 25(2):277-283. https://doi.org/10.1038/s41591-018-0304-3

15. Goeral K, Hauck A, Atkinson A et al (2021) Early life serum neurofilament dynamics predict neurodevelopmental outcome of preterm infants. J Neurol https://doi.org/10.1007/ s00415-021-10429-5 